\title{
Perspectives on the immunologic microenvironment of astrocytomas
}

This article was published in the following Dove Press journal:

Cancer Management and Research

29 August 2013

Number of times this article has been viewed

\author{
Iman $\mathrm{H}$ Hewedi' \\ Nehal A Radwan' \\ Lobna S Shash' \\ Tarek H Elserry ${ }^{2}$ \\ 'Departments of Pathology, \\ ${ }^{2}$ Neurosurgery, Faculty of Medicine, \\ Ain Shams University, Cairo, Egypt
}

Correspondence: Iman H Hewedi Departments of Pathology, Faculty of Medicine, Ain Shams University, Ramses Street, New Faculty Building, Fifth Floor, PO Box I I566, Cairo, Egypt

Tel +2020I2 23953221

Fax +202 26329776

Email iman_hewedi@med.asu.edu.eg
Background: The microenvironment of astrocytomas includes infiltrative inflammatory cells that are dynamic in nature, possibly reflecting tumor biology. We evaluated the inflammatory cell infiltrate in astrocytic tumors aiming for a better understanding of their immunobiology.

Methods: Immunohistochemical expression of CD68, CD3, and CD20 was investigated in 21 glioblastomas, 21 anaplastic astrocytomas, 13 diffuse astrocytomas, and 18 pilocytic astrocytomas. The inflammatory infiltrate was classified based on microanatomic location as perivascular and intratumoral, and subsequently graded semiquantitatively.

Results: Perivascularly, CD68-positive infiltrate was noted in $71.4 \%$ of glioblastomas compared with $14.3 \%$ of anaplastic astrocytomas $(P=0.0001), 7.7 \%$ of diffuse astrocytomas $(P=0.0001)$, and $33.3 \%$ of pilocytic astrocytomas $(P=0.017)$. Intratumorally, $85.7 \%$ of glioblastomas exhibited CD68-positive infiltrate compared with $42.9 \%$ of anaplastic astrocytomas $(P=0.004), 38.5 \%$ of diffuse astrocytomas $(P=0.008)$, and $33.3 \%$ of pilocytic astrocytomas $(P=0.001)$. Among diffusely infiltrating astrocytomas, intratumoral CD3-positive infiltrate was only associated with glioblastoma. A CD20-positive infiltrate was only detected in the perivascular space of a single case of diffuse astrocytoma.

Conclusion: These data indicate a distinct immune profile in the glioblastoma microenvironment primarily related to the prevalence of macrophages. Thus, novel glioblastoma therapies should address this key CD68-positive population and its possible role in generating an antitumor immune response.

Keywords: inflammatory cell infiltrate, astrocytoma, glioblastoma, CD68, CD3

\section{Introduction}

Malignant gliomas are heterogeneous, diffuse, and highly infiltrating by nature. Despite wide surgical resection and improvements in radiotherapy and chemotherapy, these tumors are still incurable and the prognosis of patients with glioblastoma remains extremely dismal. ${ }^{1}$

The aggressive nature of glioblastoma is closely related to its complex pathophysiology, in particular, its ability to disturb the antitumoral immune response. ${ }^{2}$ Although the central nervous system is conventionally regarded as "immunologically privileged", being shielded by the blood-brain barrier, recent studies suggest that inflammatory cells are an important component of the glioma microenvironment. ${ }^{3,4}$ Consequently, triggering the immune response in these tumors is theoretically an attractive treatment strategy because even invading scattered tumor cells can be selectively targeted without damaging the normal brain. ${ }^{5}$ 
Innate immunity is the initial antigen-nonspecific response that results in rapid production of effector cytokines that in turn mature and activate antigen-presenting cells. One of the most important prerequisites for the immune activation of T-cell responses is the presence of functional antigenpresenting cells that process and present tumor antigens, secrete specific cytokines and chemokines, and abundantly express costimulatory molecules essential for initiating and/ or propagating tumor-directed T-cells. In gliomas, antigenpresenting cells candidates have included microglia, monocyte/macrophages, B-lymphocytes, and dendritic cells. ${ }^{6}$

The location of the immune cells may also affect tumorhost interaction and may influence tumor growth. Perivascular inflammatory infiltrates may differ from intratumoral T-cell infiltrates due to differences in the proximity of the latter to the tumor cell. The intratumoral T-cell infiltrates may be associated with a more direct functional effect on tumor cells. ${ }^{7}$

The present study was motivated by the still limited number of detailed investigations addressing the inflammatory infiltrate in surgically retrieved human astrocytic tumors. This study aims to assess the overall features of the inflammatory infiltrate in diffuse astrocytomas, anaplastic astrocytomas, glioblastomas, and pilocytic astrocytomas. We evaluated cells positive for CD68, CD3, and CD20 in both perivascular and intratumoral areas for all studied astrocytomas in an attempt to explore the possibility of a specific immune cell profile pattern among these tumors.

\section{Materials and methods}

\section{Tissue specimens}

This retrospective study was conducted on 73 cases of astrocytomas, including 21 glioblastomas, 21 anaplastic astrocytomas (grade III), 13 diffuse astrocytomas (grade II), and 18 pilocytic astrocytomas. All cases were retrieved from the archives of the Department of Pathology, Ain Shams University Specialized Hospital, Cairo, Egypt. All grade II diffuse astrocytomas received between May 2007 and January 2013 were included, while comparable numbers of glioblastomas, anaplastic astrocytomas, and pilocytic astrocytomas received during the same period were selected via random sampling. All tissue specimens were obtained from the initial surgery of patients prior to any adjuvant treatment.

The cases included were selected to have sufficient representative tissues for evaluation following comprehensive neuropathologic review based on the current World Health Organization classification. ${ }^{8}$ The study was carried out with full local ethics approval.

\section{Immunohistochemistry}

Immunohistochemical staining was carried out on serial $4 \mu \mathrm{m}$ sections of formalin-fixed paraffin-embedded tissue using a standard labeled streptavidin-biotin-peroxidase complex technique, and diaminobenzidine served as the chromogen. Staining was performed applying a mouse monoclonal antibody against CD68 (Cell Marque, Rocklin, CA, USA, catalog number 168M-98, ready-to-use), a rabbit polyclonal antibody against CD3 (Cell Marque, catalog number 103A-76, dilution 1:500), and a mouse monoclonal antibody against CD20 (Lab Vision, Kalamazoo, MI, USA, catalog number MS-340 S0, dilution 1:250). Lymph nodal tissues were used as a positive control, while a negative control was achieved using the same tissues but omitting the primary antibody.

\section{Immunohistochemical analysis}

Immunohistochemical assessment was performed on a semiquantitative basis using a four-tier scale according to the scoring system described by Yang et al. ${ }^{4}$ The scoring was performed in ten consecutive high-powered fields (HPFs) and in three separate regions of the tissue (a total of $\left.30 \times 0.56 \mathrm{~mm}^{2}\right)$. Inflammatory infiltrates within the tumor tissue (intratumoral) were evaluated separately from inflammatory cells within the Virchow-Robin spaces (perivascular). The regions of tissue with the highest degree of inflammation (not associated with necrosis or hemorrhage) were selected for analysis. The intratumoral infiltrate was scored $0-3$ using the following scheme: 0 , rare infiltrate of $<5$ cells per ten HPFs; 1 , focal infiltrate of 5-20 cells per ten HPFs; 2 , intermediate infiltrate of $20-100$ cells per ten HPFs; and 3, extensive infiltrate of $>100$ cells per ten HPFs. The perivascular inflammatory cell infiltrate was analyzed for at least 20 small vessels per each ten HPFs and scored 0-3 as follows: 0 , rare infiltrate of less than one cell per vessel; 1, focal infiltrate of $2-5$ cells per vessel; 2 , intermediate infiltrate of 5-20 cells per vessel; and 3, extensive infiltrate of $>20$ cells per vessels. For all immunostains, 0 and $1+$ are regarded as negative expression, while $2+$ and $3+$ are regarded as positive expression.

\section{Statistical analysis}

Analysis was done using Statistical Package for Social Science version 15.0.1 software (SPSS Inc., Chicago, IL, USA). The Chi-square test and Fisher's exact test were used as appropriate to compare the two study groups. The McNemar test was used to assess the statistical difference of intratumoral and perivascular localization for inflammatory infiltrate among any studied group. $P$-values $<0.05$ were 
considered to be statistically significant and those $<0.01$ were highly significant.

\section{Results}

The age and sex distribution of the study cases are summarized in Table 1 . We critically assessed all astrocytomas for the presence of tumor-associated microglia/macrophages, T-cells, and B-cells to determine the overall features of the inflammatory infiltrates in these tumors (Table 2 and Figures 1 and 2).

\section{CD68-positive cells}

The density of CD68-positive cells that represented the microglia/macrophage population was significantly higher in glioblastoma compared with all other groups studied. In the perivascular space, CD68-positive infiltrate was noted in $71.4 \%$ of glioblastomas compared with $14.3 \%$ of anaplastic astrocytomas $(P=0.0001), 7.7 \%$ of diffuse astrocytomas $(P=0.0001)$, and $33.3 \%$ of pilocytic astrocytomas $(P=0.017)$. In the intratumoral compartment, $85.7 \%$ of glioblastomas showed CD68-positive infiltrate compared with $42.9 \%$ of anaplastic astrocytomas $(P=0.004), 38.5 \%$ of diffuse astrocytomas $(P=0.008)$, and $33.3 \%$ of pilocytic astrocytomas $(P=0.001)$.

The CD68 infiltrate was statistically similar between pilocytic astrocytomas and diffuse astrocytomas in both perivascular and intratumoral regions $(P=0.191$ and $P=1.00$, respectively). The same observation was made on comparing pilocytic astrocytomas with anaplastic astrocytomas regarding CD68 infiltrate whether perivascular or intratumoral ( $P=0.255$ and $P=0.542$, respectively). Lastly, no significant difference was noted between diffuse astrocytomas and anaplastic astrocytomas regarding the CD68-positive population in the perivascular region $(7.7 \%$ versus $14.3 \%, P=1.00)$ or in the intratumoral region $(38.5 \%$ versus $42.9 \%, P=0.800)$.
The spatial distribution of the CD68-positive infiltrate as perivascular or intratumoral for each tumor group studied was carefully addressed (Table 2). CD68-positive infiltrate was more prevalent in the intratumoral region than in the perivascular space among anaplastic astrocytomas $(42.9 \%$ versus $14.3 \%, P=0.31, \mathrm{McNemar}$ test).

As a final point, we compared intratumoral and perivascular CD68-positive infiltrate in pilocytic astrocytomas, anaplastic astrocytomas, and glioblastomas between adult and pediatric cases. The only age-related statistically significant difference was noted for the perivascular CD68 infiltrate among glioblastomas. Fifteen of 18 adult glioblastomas (83.3\%) had a positive perivascular CD68 infiltrate, while all three pediatric glioblastomas had a negative perivascular CD68 ( $P=0.015$, Fisher's exact test).

\section{CD3-positive cells}

Among diffusely infiltrating astrocytomas, perivascular T-cell lymphocytic infiltrate was noted in only three cases of glioblastomas (14.3\%) and a single case of diffuse astrocytoma (7.7\%). Intratumorally, no CD3-positive infiltrate was observed in any of the diffuse astrocytomas or anaplastic astrocytomas, while $28.6 \%$ of glioblastomas showed a positive intratumoral CD3 infiltrate. This relative prevalence of an intratumoral CD3-positive infiltrate among glioblastomas compared with anaplastic astrocytomas was statistically significant $(28.6 \%$ versus $0 \%, P=0.021)$.

There was no statistically significant difference regarding perivascular CD3 infiltrate in glioblastomas compared with pilocytic astrocytomas $(P=0.235)$. Glioblastomas and pilocytic astrocytomas also revealed comparable intratumoral CD3 infiltrate, being $28.6 \%$ in the former and $33.3 \%$ in the latter $(P=0.748)$.

The intratumoral CD3 infiltrate was significantly higher in pilocytic astrocytomas compared with either diffuse astrocytomas $(33.3 \%$ versus $0 \%, P=0.28)$ or anaplastic

Table I Age and sex distribution among the astrocytomas studied $(n=73)$

\begin{tabular}{|c|c|c|c|c|}
\hline & $\begin{array}{l}\text { Pilocytic astrocytoma } \\
(n=18)\end{array}$ & $\begin{array}{l}\text { Diffuse astrocytoma } \\
(n=13)\end{array}$ & $\begin{array}{l}\text { Anaplastic astrocytoma } \\
(n=2 I)\end{array}$ & $\begin{array}{l}\text { Glioblastoma } \\
(n=2 I)\end{array}$ \\
\hline \multicolumn{5}{|l|}{ Age } \\
\hline Range (years) & $4-37$ & $32-64$ & II-58 & II-57 \\
\hline Mean \pm standard deviation & $16.9 \pm 12.8$ & $45.7 \pm 11.1$ & $38 \pm 16.7$ & $42 \pm 15.4$ \\
\hline \multicolumn{5}{|c|}{ Pediatric ( $\leq 19$ years) versus adult cases } \\
\hline Pediatric, n (\%) & $12(66.6)$ & $0(0)$ & $6(28.5)$ & $3(14.3)$ \\
\hline Adult, n (\%) & $6(33.3)$ & $13(100)$ & I5 (7I.4) & I 8 (85.7) \\
\hline \multicolumn{5}{|l|}{ Sex } \\
\hline Male, n (\%) & $3(16.7)$ & II (84.6) & I5 (7I.4) & I5 (7I.4) \\
\hline Female, n (\%) & $15(83.3)$ & $2(15.4)$ & $6(28.6)$ & $6(28.6)$ \\
\hline
\end{tabular}


Table 2 Distribution of CD68 and CD3 infiltrate in all astrocytomas studied $(n=73)$

\begin{tabular}{|c|c|c|c|c|c|c|c|c|c|c|}
\hline & \multicolumn{4}{|c|}{ Perivascular } & \multicolumn{4}{|c|}{ Intratumoral } & \multirow[t]{3}{*}{$P *$} & \multirow[t]{3}{*}{ Sig } \\
\hline & \multicolumn{2}{|c|}{ Negative } & \multicolumn{2}{|c|}{ Positive } & \multicolumn{2}{|c|}{ Negative } & \multicolumn{2}{|c|}{ Positive } & & \\
\hline & $\mathbf{n}$ & $\%$ & $\mathbf{n}$ & $\%$ & $\mathbf{n}$ & $\%$ & $\mathbf{n}$ & $\%$ & & \\
\hline \multicolumn{11}{|c|}{ Pilocytic astrocytoma $(\mathrm{n}=18)$} \\
\hline CD68 & 12 & 66.7 & 6 & 33.3 & 12 & 66.7 & 6 & 33.3 & 1.00 & NS \\
\hline CD3 & 18 & 100.0 & 0 & 0.0 & 12 & 66.7 & 6 & 33.3 & 0.033 & $\mathrm{~S}$ \\
\hline \multicolumn{11}{|c|}{ Diffuse astrocytoma $(n=13)$} \\
\hline CD68 & 12 & 92.3 & 1 & 7.7 & 8 & 61.5 & 5 & 38.5 & 0.125 & NS \\
\hline CD3 & 12 & 92.3 & 1 & 7.7 & 13 & 100.0 & 0 & 0.0 & 1.00 & NS \\
\hline \multicolumn{11}{|c|}{ Anaplastic astrocytoma $(\mathrm{n}=2 \mathrm{I})$} \\
\hline CD68 & 18 & 85.7 & 3 & 14.3 & 12 & 57.1 & 9 & 42.9 & 0.031 & $S$ \\
\hline CD3 & 21 & 100.0 & 0 & 0.0 & 21 & 100.0 & 0 & 0.0 & - & - \\
\hline \multicolumn{11}{|c|}{ Glioblastoma $(\mathrm{n}=2 \mathrm{I})$} \\
\hline CD68 & 6 & 28.6 & 15 & 71.4 & 3 & 14.3 & 18 & 85.7 & 0.250 & NS \\
\hline CD3 & 18 & 85.7 & 3 & 14.3 & 15 & 71.4 & 6 & 28.6 & 0.250 & NS \\
\hline
\end{tabular}

Note: *McNemar test.

Abbreviations: S, statistically significant; NS, not statistically significant.

astrocytomas (33.3\% versus $0 \%, P=0.006)$. Among pilocytic astrocytomas, CD3-positive infiltrate was more prevalent in the intratumoral compartment than in the perivascular space (33.3\% versus $0 \%, P=0.033$, Table 2 ). Age was shown not to influence $\mathrm{CD} 3$ infiltrate either intratumorally or perivascularly among the astrocytoma groups studied.
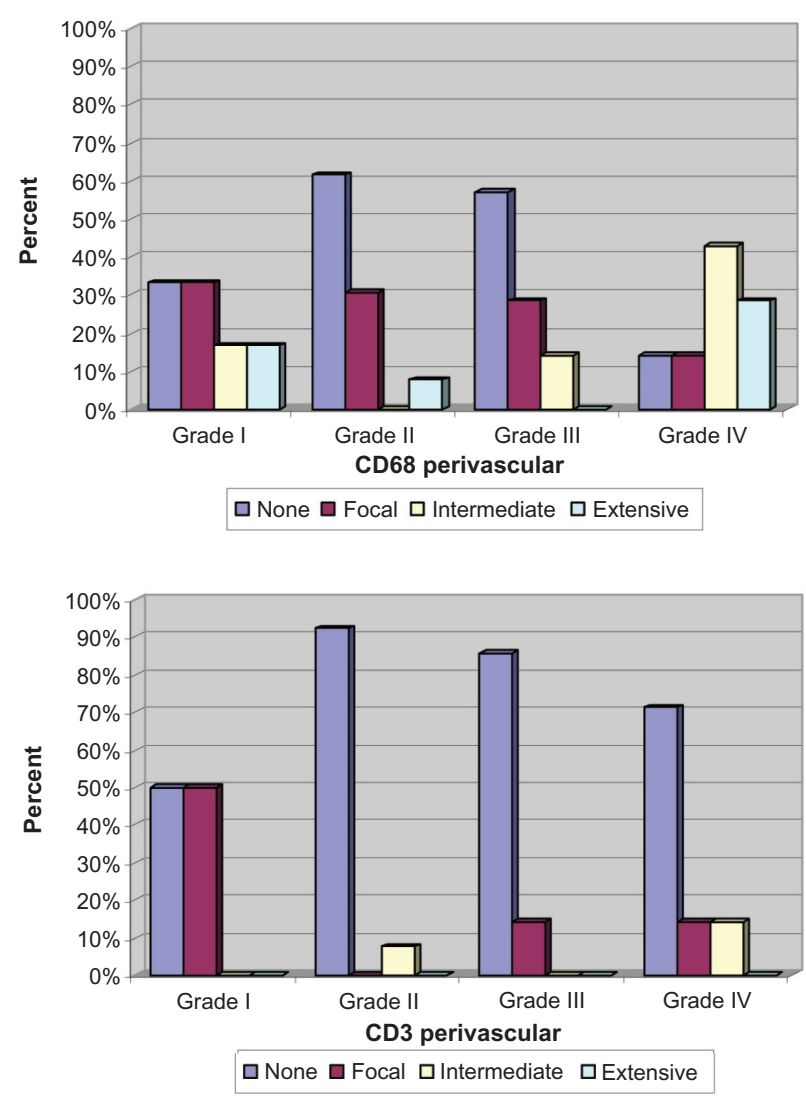

\section{CD20-positive cells}

The astrocytoma microenvironment appeared to be almost entirely shielded from B-lymphocytic infiltration. No intratumoral CD20-positive infiltrate was noted in any of the cases studied. Perivascularly, only a single case of diffuse astrocytoma showed a CD20-positive infiltrate.
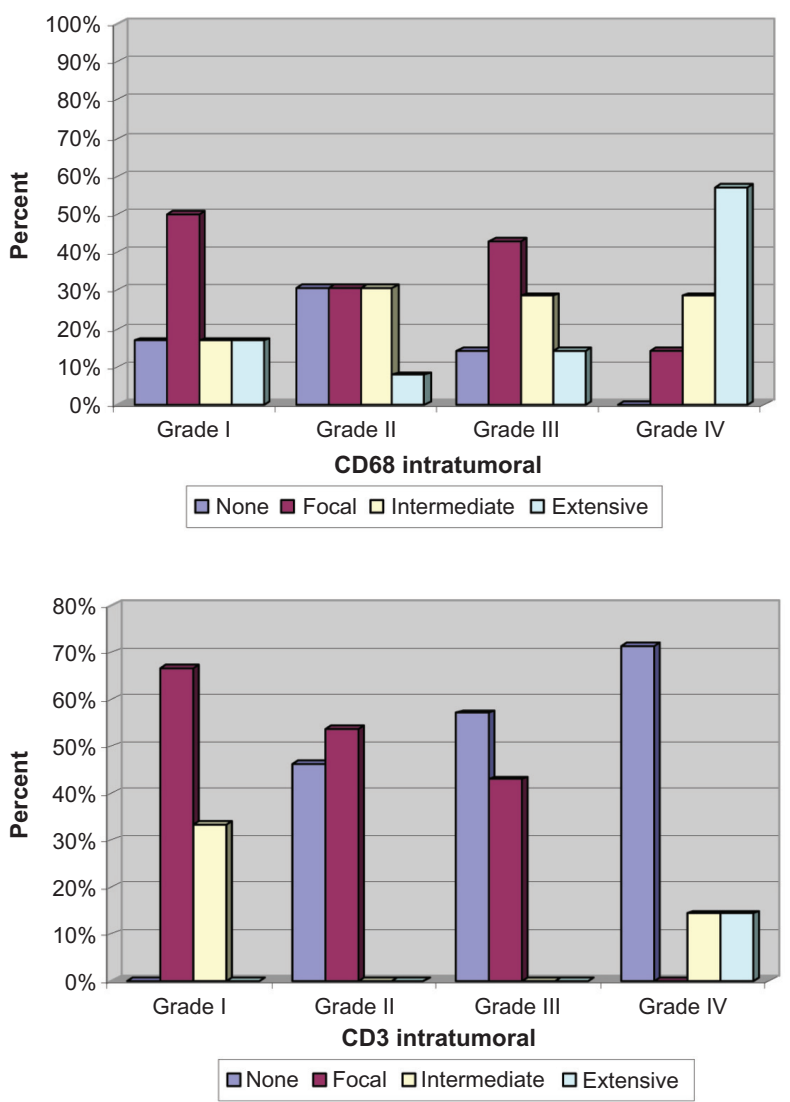

Figure I Data for semiquantitative assessment of inflammatory infiltrate among astrocytomas. 


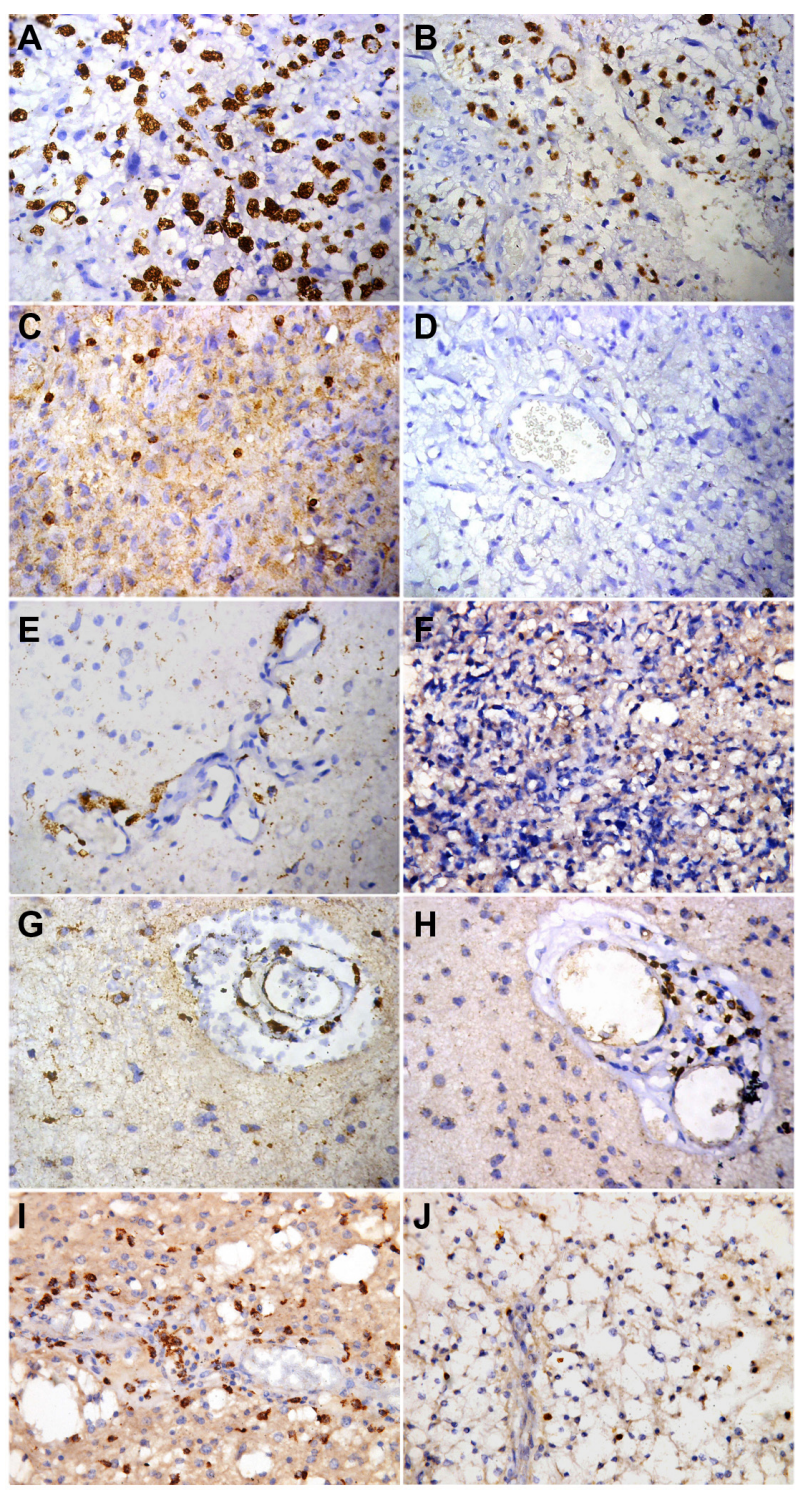

Figure 2 Synopsis of immunohistochemical markers of inflammatory infiltrate in astrocytomas. Glioblastomas: CD68 intratumoral and perivascular immunoreactivity (A and B), CD3-positive intratumoral infiltrate (C), and negative CD20 immunoreactivity (D). Anaplastic astrocytomas: CD68-positive perivascular infiltrate (E), and negative CD3 infiltrate (F). Diffuse astrocytomas: perivascular CD68 immunoreactivity (G), and CD3 perivascular infiltrate as exceptionally noted in a single case $(\mathbf{H})$. Pilocytic astrocytomas: perivascular CD68 infiltrate (I), and intratumoral CD3 infiltrate (J), (immunoperoxidase, original magnification 400×).

\section{Discussion}

The concept that inflammatory cells represent a pivotal component of the glioma microenvironment has emerged in response to a growing body of evidence..$^{2,6,9}$ This study included a comprehensive analysis of CD68, CD3, and CD20positive cells in all grades of diffusely infiltrating astrocytomas in addition to pilocytic astrocytomas, while addressing their district distribution as perivascular and intratumoral.

It has been demonstrated that tumor-associated microglia/ macrophages are the largest immune cell population in infiltrating human glioma tissue. ${ }^{10,11}$ These cells produce cytokines and signals that promote glioma growth, invasion, and angiogenesis. ${ }^{12-14}$ It has also been reported that tumor-associated microglia/macrophages likely prevent T-cell expansion in the microenvironment of such tumors. ${ }^{6}$ Yang et $\mathrm{al}^{4}$ have investigated CD68 infiltrate in glioblastomas and pilocytic astrocytomas and reported a higher density of macrophages in glioblastomas. Yi et $\mathrm{al}^{3}$ reported that the higher the histologic grade of diffusely infiltrating astrocytoma, the greater the number of infiltrating tumor-associated microglia/macrophages in tumor tissue. In this study, CD68positive infiltrate was higher in glioblastomas compared with anaplastic astrocytomas, diffuse astrocytomas, and pilocytic astrocytomas. We found that increased tumorassociated microglia/macrophages was characteristic for the glioblastoma microenvironment because a significantly lower density of CD68-positive infiltrate was noted among all the remaining astrocytic tumor groups studied. This distinct increase in tumor-associated microglia/macrophages in the glioblastoma microenvironment is suggested to be related to either a final step in the progression of astrocytoma from anaplastic astrocytoma to glioblastoma, or to a primary glioblastoma pathway. This noted boost in CD68-positive infiltrate among glioblastomas is unlikely to be passively associated with blood brain barrier breakdown, or we would expect a similar increase in CD20+ B-cells and other blood elements seen in the peripheral blood. Therefore, we infer that recruitment of tumor-associated microglia/macrophages in glioblastomas is an active process related to tumor-derived chemoattractants expressed in such tumors, which was also pointed out in previous reports. ${ }^{15,16}$

With the exception of the relative increase of the intratumoral as opposed to perivascular CD68-positive infiltrate seen in anaplastic astrocytomas, this study demonstrates a comparable distribution of macrophages in the perivascular and intratumoral regions. Yang et $\mathrm{al}^{4}$ also noted similar perivascular and intratumoral CD68-positive macrophages in glioblastomas. In contrast, the majority of tumor-associated microglia/macrophages were distributed around blood vessels in a study reported by Yi et al, suggesting a bidirectional interaction between the endothelium and microglia/ macrophages. ${ }^{3}$

Many investigators have reported the presence of glioma T-cell infiltrates in tissue from patients, suggesting generation of a spontaneous antitumor immune response. Further, they suggest that the role of infiltrating T-cells in gliomas may be critical for the design of future immunotherapies. ${ }^{5,17}$ Therefore, we investigated CD3 T-cell infiltrate in astrocytic 
tumors of all grades. We noted that CD3-positive infiltrate was comparable between glioblastomas and pilocytic astrocytomas in both the perivascular and intratumoral compartments, with slightly more intratumoral pilocytic CD3-positive infiltrate. These findings are in parallel with the observations made by Yang et al. ${ }^{4}$ The almost entirely negative CD3-positive infiltrate in diffuse astrocytomas and anaplastic astrocytomas has raised doubt that the infiltrating CD3 cells noted in glioblastomas and pilocytic astrocytomas might arise merely from a disturbed blood-brain barrier. An interesting study by Lohr et $\mathrm{al}^{5}$ in 2011 also hypothesized that leaky vessels, which typically occur in glioblastoma with the onset of angiogenesis in the context of diffusely infiltrating astrocytomas, facilitate T-cell transmigration as compared with lower grade tumors. They based their hypothesis on the parallel positive staining of both plasma protein fibrinogen and CD3 infiltrate while considering that the former cannot diffuse into the tumor except in the presence of leaky vessels. In contrast, other researchers have assumed immune recognition of gliomas by T-lymphocytes based on the difference between the noted ratio of CD4+ to CD8+ cells within the tumor tissue and the peripheral blood. ${ }^{6}$ Although our study assessed T-lymphocytic infiltrate using CD3 without further subtyping, we are still able to conclude that this CD3-positive infiltration does not represent just leakage but rather an active process based on the absence of accompanying CD20-positive B-lymphocytes and other blood elements, ie, granulocytes and erythrocytes.

Lastly, this study investigated the possible effect of patient age on the immunologic microenvironment of the astrocytomas studied, as hypothesized by Driggers et al. ${ }^{18}$ Zhang et al ${ }^{19}$ postulated an antigenic difference between pediatric and adult glioma patients upon studying 16 glioblastomas, four low-grade astrocytomas, 10 juvenile pilocytic astrocytomas, and seven ependymomas. These researchers stated that pediatric brain tumors express fewer tumor antigens when compared with adult glioblastomas, and that glioblastomas derived from adults appeared very antigenic. In this study, perivascular CD68 infiltrate in glioblastomas was only noted in adult patients, which is in line with the notion that adult glioblastoma is more antigenic relative to pediatric glioblastoma.

In conclusion, this study offers a detailed morphologic analysis of the immune cell infiltrate in all grades of diffusely infiltrating astrocytomas as well as pilocytic astrocytomas. It provides an insightful analysis regarding the variation in inflammatory infiltrate between these astrocytic tumors yet it lacked proposing clear mechanisms to explain the distinct profile noted in glioblastomas. Thus, the distinct boosting of macrophages noted in glioblastomas indicates that novel therapies for these aggressive neoplasms should address the CD68-positive population and its possible role in generating an antitumor immune response.

Further larger and more comprehensive studies addressing the functional status, biological significance, and regulatory pathways for macrophages and T-lymphocytes in infiltrating astrocytomas are mandatory. The T-cell subpopulations, ie, CD4, CD8, and regulatory T-lymphocytes, should be thoroughly investigated in various grades of human astrocytic tumor tissue along with their relative spatial distribution (perivascular or intratumoral), to characterize their possible role in the microenvironment of these tumors.

\section{Disclosure}

The authors declare that they have no competing interests in this work.

\section{References}

1. Auffinger B, Thaci B, Nigam P, Rincon E, Cheng Y, Lesniak MS. New therapeutic approaches for malignant glioma: in search of the Rosetta stone. F1000 Med Rep. 2012;4:18.

2. Sonabend AM, Rolle CE, Lesniak MS. The role of regulatory T cells in malignant glioma. Anticancer Res. 2008;28(2B):1143-1150.

3. Yi L, Xiao H, Xu M, et al. Glioma-initiating cells: a predominant role in microglia/macrophages tropism to glioma. J Neuroimmunol. 2011;232: $75-82$.

4. Yang I, Han SJ, Sughrue ME, Tihan T, Parsa AT. Immune cell infiltrate differences in pilocytic astrocytoma and glioblastoma: evidence of distinct immunological microenvironments that reflect tumor biology. J Neurosurg. 2011;115:505-511.

5. Lohr J, RatliffT, Huppertz A, et al. Effector T-cell infiltration positively impacts survival of glioblastoma patients and is impaired by tumorderived TGF- $\beta$. Clin Cancer Res. 2011;17:4296-4308.

6. Hussain SF, Yang D, Suki D, Aldape K, Grimm E, Heimberger AB. The role of human glioma-infiltrating microglia/macrophages in mediating antitumor immune responses. Neuro Oncol. 2006;8:261-279.

7. Willis SN, Mallozzi SS, Rodig SJ, et al. The microenvironment of germ cell tumors harbors a prominent antigen-driven humoral response. J Immunol. 2009;182:3310-3317.

8. Louis DN, Ohgaki H, Wiestler OD, Cavenee WK. WHO Classification of Tumors of the Central Nervous System. Lyon, France: International Agency for Research on Cancer; 2007.

9. Dunn GP, Dunn IF, Curry WT. Focus on TILs: prognostic significance of tumor infiltrating lymphocytes in human glioma. Cancer Immun. 2007;7:12.

10. Graeber MB, Scheithauer BW, Kreutzberg GW. Microglia in brain tumors. Glia. 2002;40:252-259.

11. Watters JJ, Schartner JM, Badie B. Microglia function in brain tumors. J Neurosci Res. 2005;81:447-455.

12. Zeisberger SM, Odermatt B, Marty C, Zehnder-Fjällman AH, Ballmer-Hofer K, Schwendener RA. Clodronate-liposome-mediated depletion of tumour-associated macrophages: a new and highly effective antiangiogenic therapy approach. Br J Cancer. 2006;95:272-281.

13. Wesolowska A, Kwiatkowska A, Slomnicki L, et al. Microglia-derived TGF-beta as an important regulator of glioblastoma invasion - an inhibition of TGF-beta-dependent effects by shRNA against human TGF-beta type II receptor. Oncogene. 2008;27:918-930. 
14. Hong TM, Teng LJ, Shun CT, Peng MC, Tsai JC. Induced interleukin-8 expression in gliomas by tumor-associated macrophages. J Neurooncol. 2009;93:289-301.

15. Kerber M, Reiss Y, Wickersheim A, et al. Flt-1 signaling in macrophages promotes glioma growth in vivo. Cancer Res. 2008;68: 7342-7351.

16. Held-Feindt J, Hattermann K, Müerköster SS, et al. CX3CR1 promotes recruitment of human glioma-infiltrating microglia/macrophages (GIMs). Exp Cell Res. 2010;316:1553-1566.
17. Ueda R, Low KL, Zhu X, et al. Spontaneous immune responses against glioma-associated antigens in a long term survivor with malignant glioma. J Transl Med. 2007;5:68.

18. Driggers L, Zhang JG, Newcomb EW, et al. Immunotherapy of pediatric brain tumor patients should include an immunoprevention strategy: a medical hypothesis paper. J Neurooncol. 2010;97:159-169.

19. Zhang JG, Kruse CA, Driggers L, et al. Tumor antigen precursor protein profiles of adult and pediatric brain tumors identify potential targets for immunotherapy. J Neurooncol. 2008;88:65-76.

\section{Publish your work in this journal}

Cancer Management and Research is an international, peer-reviewed open access journal focusing on cancer research and the optimal use of preventative and integrated treatment interventions to achieve improved outcomes, enhanced survival and quality of life for the cancer patient The journal welcomes original research, clinical \& epidemiological studies, reviews \& evaluations, guidelines, expert opinion \& commentary, case reports \& extended reports. The manuscript management system is completely online and includes a very quick and fair peerreview system, which is all easy to use. Visit http://www.dovepress.com/ testimonials.php to read real quotes from published authors.

Submit your manuscript here: http://www.dovepress.com/cancer-management-and-research-journal 